\title{
Rezago en asignaturas de contenido matemático en estudiantes de último año: analizando resultados de colegios en territorios indígenas y del Gran Ảrea Metropolitana de Costa Rica ${ }^{[1]}$
}

\author{
Moreira-Mora, Tania Elena; Zamora-Araya, José Andrey; Smith-Castro, Vanessa; Montero-Rojas, Eiliana \\ Rezago en asignaturas de contenido matemático en estudiantes de último año: analizando resultados de \\ colegios en territorios indígenas y del Gran Área Metropolitana de Costa Rica [1] \\ Revista Educación, vol. 45, núm. 1, 2021 \\ Universidad de Costa Rica, Costa Rica \\ Disponible en: http://www.redalyc.org/articulo.oa?id=44064134016 \\ DOI: https://doi.org/10.15517/revedu.v45i1.41317
}

Esta obra está bajo una Licencia Creative Commons Atribución-NoComercial-SinDerivar 3.0 Internacional. 


\title{
Rezago en asignaturas de contenido matemático en estudiantes de último año: analizando resultados de colegios en territorios indígenas y del Gran Área Metropolitana de Costa Rica ${ }^{[1]}$
}

\author{
Math Difficulties in High School Seniors: Analysis of Results from High Schools in Costa Rica's Indigenous \\ Territories and the Greater Metropolitan Area
}

Tania Elena Moreira-Mora

Instituto Tecnológico de Costa Rica, Costa Rica

tmoreira@itcr.ac.cr

(DD http://orcid.org/0000-0002-8955-0804

José Andrey Zamora-Araya

Universidad Nacional, Costa Rica

jzamo@una.ac.cr

(D) http://orcid.org/0000-0001-6050-5850

Vanessa Smith-Castro

Universidad de Costa Rica, Costa Rica

vanessa.smith@ucr.ac.cr

(iD http://orcid.org/0000-0001-6348-4223

Eiliana Montero-Rojas

Universidad de Costa Rica, Costa Rica

eiliana.montero@ucr.ac.cr

D http://orcid.org/0000-0002-6770-792X
DOI: https://doi.org/10.15517/revedu.v45i1.41317

Redalyc: http://www.redalyc.org/articulo.oa?id=44064134016 
The School Effectiveness Framework was used to examine three dimensions: socio-demographic characteristics, attitudes towards Math and social beliefs about Math contexts with the objective of exploring the predictive value of variables associated with difficulties in Math-related subjects for high school seniors (last year students) in Indigenous territories and the Greater Metropolitan Area of Costa Rica. The is a cross-sectional and correlational study based on a sample size of 356 students who answered four questionnaires: The Math Self-Efficacy Scale, Math Usefulness, Sexist Beliefs and Math-Gender Stereotypes. Multilevel regression models were calculated using two dependent variables: a) a count indicator for difficulties in Math-related subjects and, b) a variable identifying students who needed to take a remedial test to pass the subject. Results reveal that Math SelfEfficacy was the principal predictor of difficulties in both populations. Benevolent Sexism and Equity were also relevant only for BMA-age high school students. The explicative value of the predictors in both dependent variables is considerably higher for BMA schools. Despite contextual differences, it can be concluded that that Math Self-efficacy is a relevant predictor for indicators related to Math difficulties and its use should be promoted in classrooms. However, more research is needed, specifically in indigenous territories, to help reduce factors that are causing students to lag behind in Math in this specific population group.

KEYworDs: Math Difficulties, Indigenous Territories in Costa Rica, Attitudes, Social Beliefs, Multilevel Regression Models.

\section{INTRODUCCIÓN}

El tema de los factores relacionados al rendimiento académico en matemáticas en la educación secundaria ha sido ampliamente investigado en el ámbito nacional e internacional, desde diversas posturas teóricas y metodológicas, considerando factores intrínsecos del sujeto como motivación, habilidades cognitivas, estrategias de aprendizaje, entre otros; así como factores extrínsecos relativos al contexto familiar, educativo y social. En América Latina los estudios de eficacia escolar han tenido una gran trayectoria en la investigación educativa. Para Gamazo, Martínez, Olmos y Rodríguez (2018), los factores más comúnmente estudiados sobre el rendimiento son de diversa naturaleza, entre los que destacan los factores de entrada relacionados con el nivel socio-económico, género, recursos educativos, lengua materna, nivel educativo de padres y otros; los factores de proceso o no contextuales concernientes a hábitos de estudio, metodología docente, motivación, autoeficacia y el autoconcepto entre otros; y el producto relativo al rendimiento académico.

Estas investigaciones educativas sobre factores asociados al aprendizaje se han abordado tradicionalmente desde tres grandes perspectivas teóricas, según Cornejo y Redondo (2007) en: la reestructuración enfocada en aquellos procesos de aprendizaje desde el constructivismo; la segunda, centrada en una visión más cultural de la dinámica de las instituciones educativas y la tercera sobre la eficacia escolar. Para estos autores la mayoría de los estudios de factores asociados al aprendizaje en América Latina se han enmarcado en la tradición de la productividad educativa, cuya principal limitación es que se basan en un restringido grupo de variables predictivas, que no incluyen variables de proceso referidas a la vida social y cultural de las instituciones.

No obstante, los estudios en esta última línea de investigación han crecido en la región latinoamericana, debido a que la eficacia escolar es un reflejo de las inequidades en materia educativa que enfrenta el estudiantado perteneciente a los sectores más desfavorecidos de la sociedad y, al ser América Latina una de las regiones más desiguales del mundo, es comprensible el interés en esta temática (Woitschach, Fernández, Martínez y Muñiz, 2017). Es así como los estudios en esta área se remontan a las conclusiones del famoso informe Coleman (Coleman, 1966) sobre la poca incidencia de la intervención escolar en el rendimiento académico, lo que propició una serie de estudios sobre el tema que han ganado popularidad, en parte, debido a la disponibilidad de fuentes de información tales como las pruebas PISA (Gamazo et al., 2018).

Desde una perspectiva integral, Martínez y Murillo (2016) entienden la enseñanza eficaz como

la acción del docente que consigue un desarrollo integral y perdurable de todos y cada uno de sus estudiantes mayor de lo que sería esperable teniendo en cuenta su rendimiento previo y la situación social, económica y cultural de las familias (p. 472).

En esta definición, los autores plantean que la investigación sobre eficacia escolar está caracterizada por la vinculación del aula y la escuela, junto con el hecho de que el resultado del aprendizaje no se circunscribe a lo meramente académico, sino también al componente socio-afectivo. 
Adicionalmente, las investigaciones han mostrado la importancia que tienen los factores económicos, sociodemográficos y culturales en el rendimiento académico, tanto en materias relacionadas con la lengua materna como numéricas, aunque para el caso de América Latina, algunas investigaciones sugieren un efecto ligeramente mayor en el área de matemáticas (Cervini, 2010; Murillo y Román, 2011; Zorrilla, 2009). También hay evidencia de que variables como el ser indígena y trabajar son relevantes en el contexto latinoamericano (UNESCO-OREALC, 2016).

Dentro la perspectiva teórica de eficacia escolar, esta investigación se ha enfocado en campo de la productividad educativa, particularmente, en el análisis del rezago académico en alguna asignatura con contenido matemático en colegios ubicados en territorios indígenas considerando tres factores: el sociodemográfico, la autoeficacia en matemáticas y las creencias sociales, con el propósito de explorar el valor predictivo de variables que podrían estar asociadas al rezago en asignaturas de contenido matemático (ciencias, química, física, biología y matemática) en estudiantes de colegios ubicados en territorios indígenas y del Gran Área Metropolitana de Costa Rica (GAM).

En estos estudios de eficacia escolar es de sumo interés la rendición de cuentas de los centros educativos, particularmente, sobre el rendimiento académico. En el caso de Costa Rica se ha dado un crecimiento en la cobertura de la educación secundaria durante el período 2010-2016, que se refleja tanto en la tasa de cobertura bruta de 84,8\% a 95,9\%, como en la tasa neta de 67,7\% a 73,3\% (Programa Estado de la Nación, 2017). Sin embargo, aún persisten problemas de eficiencia interna del sistema que limitan el logro de avances mayores, particularmente, en los territorios indígenas. Según el Instituto Nacional de Estadística y Censo (INEC, 2013), en Costa Rica existen 24 territorios indígenas donde residen el 2,42\% de la población que se declara indígena. Estas personas residen, mayoritariamente, en zonas aisladas y con un nivel muy bajo en el Índice de Desarrollo Social (IDS).

Con respecto al acceso al sistema escolar, señala Fernández (2018) que del total de estudiantes que cada año se matricula en colegios, menos del $1 \%$ corresponde a estudiantes que asisten a centros educativos ubicados en territorios indígenas. Según los datos del último censo, la población indígena asiste en menor medida a los colegios del país, aunque el porcentaje ha aumentado considerablemente al pasar del $42 \%$ al $72 \%$ entre los años 2000 y 2011 (INEC, 2013).

Además de la baja matrícula en territorios indígenas, también se presenta el problema del bajo porcentaje de graduados. Según el Instituto Nacional de las Mujeres (INAMU, 2006) la condición de nombramientos interinos del equipo docente en territorios indígenas y el no ser necesariamente indígenas son factores que dificultan el seguimiento del proceso de enseñanza. Por otra parte, Carvajal, Cubillo y Vargas (2017) destacan el problema de la carencia de docentes indígenas formados como tales por una institución de educación superior.

En definitiva, condiciones tales como la inestabilidad del personal docente y el insuficiente personal graduado, sumado a la ubicación de los colegios en zonas muy alejadas y poco accesibles, con escasos bienes y servicios, la baja matrícula y promoción en la enseñanza media, pueden incidir en el rezago académico del estudiantado de colegios en territorios indígenas. Como se comprueba con los datos del último censo (INEC, 2013), la población de 7 a 17 años que tiene al menos un año de rezago escolaridad es de 40,6\% en los territorios indígenas, en tanto que en el ámbito nacional es de 23,6\%.

$\mathrm{Al}$ respecto, Fernández (2018) explica que, en promedio, la promoción del país en pruebas de bachillerato ronda el 70\% (incluyendo colegios públicos, privados, subvencionados y otras ofertas del sector público), en el caso de los colegios ubicados en territorios indígenas, la promoción ha sido inferior al $50 \%$ todos los años desde el 2010. Esta diferencia se marca aún más en los resultados por asignatura, en el año 2016 mientras en los colegios públicos no indígenas la nota promedio en matemáticas fue de 58 (sobre 100), en los colegios indígenas fue de 49, lo que demuestra su rezago académico en la conclusión del bachillerato en secundaria. 


\section{ESTADO DE LA CUESTIÓN}

\section{Factores socio-demográficos}

En el contexto latinoamericano existen marcadas desigualdades sociales y económicas que limitan las oportunidades educativas para quienes se encuentran en los estratos más bajos. Al respecto, Galindo (2019) señala, a partir de los resultados de las pruebas PISA aplicadas en el 2018 a los jóvenes de 15 años en las áreas de matemáticas, lectura y ciencias, que los países de la región están a la cola del índice de inclusión social. En cuanto a las evaluaciones obtenidas por el estudiantado, se encontró que Costa Rica empeoró en lectura, se quedó igual en matemática y mejoró en ciencias. Finalmente, concluye este autor que lograr la igualdad de oportunidades es un objetivo muy complicado, no solo por los altos grados de segregación en la red educativa, la falta de materiales, las diferencias en la formación del personal docente, sino también por los contactos y códigos culturales que se vuelven menos accesibles para aquellos que no nacieron en la parte alta de la distribución de renta.

En el 2011 se realizó una investigación con el estudiantado de tres colegios indígenas, con el propósito de estudiar la posibilidad de utilizar algunas pruebas psicométricas, distintas a las utilizadas tradicionalmente para el proceso de admisión en la Universidad de Costa Rica (UCR) y del Instituto Tecnológico de Costa Rica (TEC). De acuerdo con Montero et al. (2013), a partir de los resultados de una prueba de razonamiento con figuras (PRF) y de cada una de las pruebas de admisión, se encontraron diferencias a favor de la PRF en relación con ambas pruebas de selección. La principal conclusión es que estas primeras evidencias apoyan la validez de criterio de los resultados de la PRF en el contexto de su aplicación como posible instrumento de selección complementario o alternativo para ingreso a la UCR y al TEC en este grupo de población.

$\mathrm{Al}$ respecto, Aponte (2008) demuestra que la población indígena presenta el menor nivel de escolaridad, lo cual continúa evidenciando el alto nivel de exclusión en los sistemas educativos, lo que genera obstáculos para que estos y estas estudiantes tengan acceso a la educación superior. Para este autor, las diferencias de raza, etnia y privación socioeconómica no han sido factores vinculados a la inclusión, los accesos y la igualdad de la provisión de oportunidades educativas en el nivel superior.

Con respecto a la investigación acerca de las diferencias de género, en múltiples estudios se han encontrado evidencias a favor de los hombres en el desempeño académico en las áreas de matemática y ciencias; mientras que las mujeres tienden usualmente a presentar mejores resultados en el área de lectoescritura (Lindberg, Hyde, Petersen y Linn, 2010; Reilly, Neumann y Andrews, 2019). Según la Organización para la Cooperación y el Desarrollo Económicos (OCDE) (2015), en los más recientes estudios de PISA se han encontrado, consistentemente, que las mujeres superan a los varones en lectura y en algunos de los estudios en más de 25 puntos; mientras que el rendimiento de los varones en ciencias es, en promedio, cuatro puntos más alto que el de las mujeres en 24 países y economías estudiadas. También en matemáticas los varones superan a las mujeres en ocho puntos y las diferencias resultan más grandes en Austria, Brasil, Argentina, Chile, Costa Rica, Alemania, Irlanda, Italia, Líbano y España, donde el puntaje promedio de los chicos supera al de las chicas por más de 15 puntos (OCDE, 2016).

\section{Actitudes hacia las matemáticas}

Los factores socioafectivos se han relacionado con el bajo rendimiento en la asignatura de matemática, en particular con las creencias y las actitudes hacia la materia que, en muchos casos, provocan inseguridad o rechazo por parte del estudiantado. Tales actitudes negativas hacia las matemáticas parecen tener su origen en malas experiencias previas, pues en ocasiones el estudiantado no logra comprender su contenido, lo que 
podría generar falta de confianza, desinterés, ansiedad e incluso temor hacia la materia (Casis, Rico y Castro, 2017).

Un importante número de estudios como los de Kaya y Bozdag (2016); Olivier, Archambault, De Clercq y Galand (2019); Schöber, Schütte, Köller, McElvany y Gebauer (2018) y Zamora-Araya (2020) muestran que una alta autoeficacia percibida aumenta las probabilidades de éxito académico en virtud de su asociación con la motivación del logro académico y el aprendizaje En particular, se ha observado que estudiantes con un alto sentido de autoeficacia matemática tienden a participar más en tareas desafiantes, invierten más esfuerzo en su ejecución, son más persistentes, y muestran un mejor rendimiento académico en comparación con las y los estudiantes que carecen de tanta confianza (Bong, 2001).

Otras investigaciones muestran que el agrado que siente el estudiantado por las matemáticas le permite implicarse más o menos en su estudio. Específicamente, la importancia que se le otorgue a la matemática y la utilidad que se le atribuye aparecen asociadas a la apreciación de la materia de cara al futuro y a los logros académicos actuales (Muñoz, Arias y Mato, 2018).

\section{Creencias sociales sobre el género}

La investigación alrededor de las brechas de género apunta al papel de las creencias sociales y los estereotipos culturales con las capacidades de hombres y mujeres, particularmente, en el desempeño académico y los planes vocacionales del estudiantado (Ellis, Fosdick y Rasmussen, 2016). Por ejemplo, se ha mostrado que el rendimiento de las mujeres es menor (en comparación con los hombres) cuando se activan creencias negativas acerca de las habilidades de las mujeres o su capacidad en matemáticas (Spencer, Steele y Quinn, 1999; Pennington, Heim, Levy y Larkin, 2016). La investigación indica, además, que el impacto negativo de la amenaza del estereotipo en el desempeño matemático de las mujeres puede llegar a tener efectos tan grandes como 20 puntos en el componente de matemáticas de pruebas estandarizadas como el Scholastic Aptitute Test (Walton y Spencer, 2009).

Otras investigaciones muestran que las creencias negativas y los estereotipos de género se sustentan sistemas de creencias sexistas que refuerzan los roles tradicionales de hombres y mujeres. Por ejemplo, datos recientes de Zell, Strickhouser, Lane y Teeter (2016) han demostrado que las personas que avalan creencias sexistas tienden a percibir más diferencias de género y a exagerar el tamaño de tales diferencias en varios dominios, incluyendo pruebas de matemáticas, habilidades académicas y los intereses en campos científicos.

Finalmente, otra línea de investigación muestra que los estereotipos culturales pueden afectar el desempeño en matemáticas a través de las actitudes de importantes agentes de socialización como pares, padres/madres y docentes o profesores/as (Jacobs y Eccles, 1992; Wigfield y Gladstone, 2019). Específicamente, la evidencia muestra que las niñas, cuyas habilidades en matemáticas son cuestionadas repetidamente por su entorno, desarrollan un autoconcepto matemático más bajo, menos confianza en su aptitud matemática y están menos motivadas que los niños en el dominio de las matemáticas (Brown y Leaper, 2010).

\section{REFERENTES TEÓRICO-CONCEPTUALES}

\section{Actitudes hacia la matemática}

Las actitudes hacia las matemáticas se refieren a la valoración y el disfrute de esta disciplina. La investigación indica que el constructo es de carácter multidimensional e incluye aspectos como la autoeficacia matemática, la utilidad percibida de la matemática, la ansiedad, el disfrute de la materia y las expectativas de las y los docentes, entre otros (Palacios, Arias y Arias, 2014). En general, la autoeficacia se trata del grado de 
convencimiento de que son eficaces para resolver eventualidades de la vida cotidiana (Schwarzer, Bäßler, Kwiatek, Schröder y Zhang, 1997).

La autoeficacia puede influir tanto en sentimientos como en pensamientos y acciones. Las personas con pocas expectativas tienden a mostrar baja autoestima y sentimientos negativos sobre su capacidad. En cuanto a los pensamientos, la percepción de autoeficacia facilita las cogniciones referidas a las habilidades propias, actuando estos pensamientos como motivadores de la acción. Finalmente, cuando se habla de autoeficacia se hace referencia a la eficacia percibida en una situación específica, por ejemplo, aprobar un examen de matemáticas.

La utilidad percibida de la matemática se refiere al valor dado a los conocimientos matemáticos como herramientas para la vida (Eccles y Wigfield, 2002; Wigfield y Gladstone, 2019). Según estos autores, la motivación, persistencia y logro académico están vinculados tanto a las expectativas como al valor de las tareas y materias. Específicamente, el modelo propone cuatro dimensiones del valor sobre la importancia que atribuyen las y los estudiantes a una asignatura por: a) la motivación intrínseca y el interés genuino, b) la utilidad de la asignatura para alcanzar objetivos a corto y largo plazo, c) el valor personal dado al buen desempeño y al desarrollo de una buena comprensión conceptual y d) el costo personal como la ansiedad y el miedo al fracaso o el esfuerzo necesario para lograr los objetivos del curso.

\section{Creencias sociales sobre el género}

En lo que respecta a las ideologías sexistas, actualmente se identifican dos tipos principales de sexismo: el hostil y el benevolente (Glick y Fiske, 2011). Estas ideologías sexistas pueden afectar el rendimiento a través de la activación de estereotipos culturales sobre las diferencias de género que pueden llevar a las personas a expresar conductas congruentes con los estereotipos (profecía autocumplida), o bien, a través de sus efectos sobre la autoestima, el autoconcepto, la autoeficacia o el interés en contextos matemáticos, lo que a su vez afecta el desempeño. Estas creencias hostiles y benevolentes representan ideologías distintas, pero complementarias que sustentan desigualdades de género (Glick, Wilkerson y Cuffe, 2015).

En relación con los efectos indirectos del sexismo, la investigación en el marco de la Teoría del Sexismo Ambivalente (Glick y Fiske 2011) apunta hacia la influencia del sexismo sobre los estereotipos de género, a través de la exageración de la diferenciación de género. De acuerdo con esta teoría, el sexismo hostil tiene sus raíces en la diferenciación de género competitiva (los hombres son más competentes que las mujeres); mientras que sexismo benevolente se basa en la diferenciación de género complementaria (las mujeres son puras y cálidas, y se complementan perfectamente con los hombres, que son más capaces y competentes). Se asume entonces que la exposición a actitudes sexistas hostiles y benevolentes pueden llevar a las personas a percibir grandes diferencias entre sexos en varios rasgos, incluyendo habilidades en dominios académicos, que a su vez pueden afectar la percepción de sus habilidades, así como su motivación académica (Hyde, Lindberg, Linn, Ellis y Williams, 2008; Pennington, Heim, Levy y Larkin, 2016; Zell, Strickhouser, Lane y Teeter, 2016).

\section{Procedimientos metodológicos}

Este es un estudio transversal y correlacional basado en un análisis multivariado acerca del rezago académico en asignaturas con contenido matemático. Para estimar las relaciones entre el conjunto de variables predictoras y los indicadores de rezago en Matemática se ajustaron dos modelos de regresión multinivel en cada una de las dos muestras: colegios del GAM y colegios de territorios indígenas.

El primer modelo empleó una variable dependiente métrica que representa el número de veces que el estudiantado indicó que debió tomar la prueba, para aplazados o que arrastró alguna de las asignaturas con 
contenido matemático, como parte de su historia académica. En otras palabras, en este modelo se sumaba uno al contador de rezago para las y los estudiantes que manifestaron haber presentado la prueba de aplazados y/ o arrastrado en matemática, ciencias, biología, química o física. Así, la variable tuvo un rango entre 0 y 4 . El segundo modelo de regresión multinivel estimado fue logístico, dado que su variable dependiente o criterio fue un indicador dicotómico que tomó el valor de 1 cuando el estudiantado manifestó que presentó el examen de aplazados en matemática y 0 en otro caso. Dado que se considera que la zona geográfica del colegio es una variable moderadora (o de interacción), pues las relaciones entre las variables predictoras y las dependientes podrían cambiar según ese contexto geográfico, se determinó que el enfoque de análisis multigrupo (Little, 2013) era el más adecuado. En este enfoque se estiman separadamente los modelos para cada una de las dos categorías de población y luego se comparan para concluir sobre semejanzas y diferencias a nivel sustantivo entre sus resultados.

\section{Participantes}

En el estudio participaron de manera voluntaria un total de 356 estudiantes de undécimo año provenientes de secundarias públicas: 215 de cuatro colegios de la GAM y 141 de nueve centros ubicados en territorios indígenas de los pueblos cabécar, bribri y ngöbe o guaymi. En Costa Rica, el término pueblo hace referencia a elementos culturales, mientras que los territorios a un ámbito geográfico destinado a que las poblaciones indígenas puedan desarrollarse según sus costumbres y tradiciones (INEC, 2013). De este modo, el pueblo cabécar se distribuye en ocho territorios, los bribris en cuatro y los ngöbes en cinco. De acuerdo con Fuentes (2011), las condiciones más desfavorables se encuentran en los territorios y pueblos indígenas bribris, ngöbes y cabécares.

Esta muestra estuvo conformada por $50,8 \%$ de hombres y $49,2 \%$ de mujeres, cuyas edades oscilaban entre los 16 y 33 años. Específicamente, en esta variable el estudiantado de la GAM se ubicó entre los 16 y 19 años, con una media de 16,64 y una desviación de 0,66. Mientras que en los territorios indígenas el 69\% se ubicó en ese rango y el restante entre los 22 y los 33 años, con una media de 19,38 y una desviación de 3,21. Con respecto a los territorios indígenas, según el último censo (INEC, 2013), un 25,9\% de la población se reportó como no indígena. A todos los participantes se les explicó los propósitos de la investigación, las condiciones de confidencialidad, voluntariedad y se les solicitó la autorización para el acceso de sus datos.

\section{Instrumentos}

La recolección de los datos fue realizada entre el 2014 y el 2016 con la técnica de la encuesta, de manera presencial y auto administrada. Antes de la aplicación de las escalas, se realizó una adaptación al contexto costarricense a través de la aplicación de entrevistas cognitivas (Smith y Molina, 2011). Igualmente se examinaron las propiedades psicométricas básicas de cada escala, las cuales poseen una consistencia interna apropiada para los propósitos del estudio. Los cuatro instrumentos aplicados se contestaron en una escala Likert de 5 puntos de totalmente en desacuerdo a totalmente de acuerdo.

Autoeficacia matemática: se utilizaron los reactivos de la subescala de Confianza Personal para las Matemáticas, tomada de la Escala de Actitudes hacia las Matemáticas de Fennema y Sherman (1976), ampliamente utilizada a nivel internacional para medir las actitudes de las y los estudiantes ante la matemática. La escala consta de 11 reactivos. Algunos ejemplos de estos reactivos son: Estoy seguro/a de que puedo aprender matemáticas o No soy el tipo de persona a la que le va bien en matemáticas (ítem inverso). La subescala de autoeficacia matemática presentó un coeficiente de consistencia interna de 0,873 para toda la muestra. 
Utilidad de la matemática: Las creencias del estudiantado sobre la importancia y utilidad de las matemáticas en la vida futura fueron medidas a través de ítems extraídos también de la Escala de Actitudes hacia las Matemáticas de Fennema y Sherman (1976). Específicamente se utilizaron los reactivos de la subescala de Utilidad de la Matemática. Ejemplos de los reactivos son: Voy a necesitar las matemáticas para mi futuro trabajo o Dominar las matemáticas no es importante para mi futuro (ítem inverso). En el presente estudio, los reactivos presentaron un coeficiente de consistencia interna Alfa de Cronbach de 0,819 para toda la muestra.

Creencias sexistas hostiles y benevolentes: estos constructos fueron medidos a través de la adaptación latinoamericana de la Escala de Sexismo Ambivalente (ASI) (Cárdenas, Lay, González, Calderón y Alegría, 2010; Glick y Fiske, 1996). La ASI es una medida directa del sexismo hostil y benevolente compuesta por 22 reactivos. Once de esos ítems miden sexismo hostil (Muchas mujeres interpretan comentarios y acciones inocentes como sexistas); mientras que otros once miden sexismo benevolente (Las mujeres, en comparación con los hombres, tienden a tener una mayor sensibilidad moral). Para el presente estudio, los reactivos de la subescala de sexismo hostil presentaron un coeficiente de consistencia interna de 0,840 ; mientras que los reactivos de sexismo benevolente presentaron un coeficiente de consistencia interna de 0,789.

Estereotipos de género en contextos matemáticos: Las creencias sobre si los hombres y mujeres poseen igual capacidad para las matemáticas fueron medidos con ítems extraídos también de Escala de Actitudes hacia las Matemáticas de Fennema y Sherman (1976). Específicamente se utilizaron los reactivos de la subescala de Percepción la Matemática como Dominio Masculino. Los análisis exploratorios de factores indicaron la pertinencia de construir dos subescalas: una con aquellos reactivos tendientes a medir la idea del dominio de un género sobre el otro (La verdad es que los hombres entienden mejor las matemáticas que las mujeres); y otra subescala con los que reflejan equidad de género (Las mujeres son tan buenas como los hombres en geometría). En el presente estudio la subescala de dominio de un género presentó un coeficiente de consistencia interna Alfa de Cronbach de 0,916; mientras que la subescala de la equidad de género presentó un coeficiente de consistencia interna de 0,714 en el total de la muestra.

\section{ANÁLISIS Y DISCUSIÓN DE RESULTADOS}

En los territorios indígenas el porcentaje de hombres fue 65\%, en contraste con el GAM, donde la mayoría del estudiantado que participó en la muestra eran mujeres (58.6\%). En cuanto a las estadísticas descriptivas de las variables incluidas en el modelo multivariado, en la Tabla 1 se resumen los datos por región y total.

TABLA 1

Estadísticas descriptivas de variables en colegios de territorios indígenas y GAM

\begin{tabular}{|c|c|c|c|}
\hline \multirow[t]{2}{*}{ Variables } & \multicolumn{2}{|c|}{ Territorio indigena $\mathrm{n}=141 \mathrm{GAM} \mathrm{n}=215$} & \multirow{2}{*}{$\begin{array}{l}\text { Total n = } 356 \\
\text { Media (D.E) }\end{array}$} \\
\hline & Media (D.E) & Media (D.E) & \\
\hline Sexo & $0,350(0,478)$ & $0,590(0,494)$ & $0,494(0,501)$ \\
\hline Edad & $19,380(3,208)$ & $16,640(0,656)$ & $17,720(2,476)$ \\
\hline Contador Rezago & $0,390(0,531)$ & $0,460(0,721)$ & $0,430(0,653)$ \\
\hline Reprobó Matemática & $0,300(0,462)$ & $0,310(0,462)$ & $0,310(0,462)$ \\
\hline Autoeficacia & $3,515(0,649)$ & $3,917(0,801)$ & $3,758(0,769)$ \\
\hline Utilidad & $3,564(0,939)$ & $3,947(0,849)$ & $3,795(0,904)$ \\
\hline Benevolente & $3,707(0,650)$ & $2,826(0,752)$ & $3,175(0,833)$ \\
\hline Hostil & $3,409(0,787)$ & $2,879(0,667)$ & $3,089(0,761)$ \\
\hline Dominio & $3,012(0,832)$ & $2,066(0,692)$ & $2,441(0,881)$ \\
\hline Equidad & $3,936(0,679)$ & $4,532(0,534)$ & $4,296(0,663)$ \\
\hline
\end{tabular}




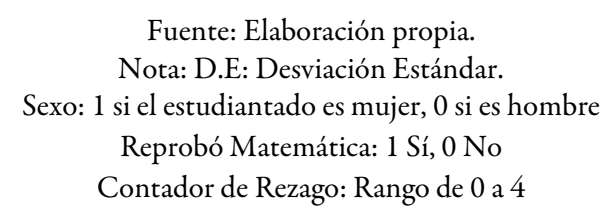

En cuanto a las variables predictoras medidas en escalas tipo Likert de 1 a 5 , el mayor puntaje se obtiene en la escala de equidad y el menor en la de dominio. Además, se nota que el estudiantado de territorios indígenas presenta mayor puntaje en las escalas de sexismo ambivalente que sus contrapartes del GAM y en las escalas de autoeficacia y utilidad los valores son similares. En cuanto a las variables dependientes, el haber presentado pruebas de aplazados cuando reprueban la asignatura de matemática muestra puntuaciones muy parecidas en ambas áreas geográficas y el contador de rezago en matemática tiene un promedio mayor en la GAM que en los territorios indígenas.

En el caso de esta investigación debe utilizarse los modelos multinivel, en vez de los modelos clásicos de regresión múltiple, dado que se trata de un muestreo de conglomerados, donde las y los estudiantes están agrupados en centros educativos, y ese factor de conglomeración, que produce mayor homogeneidad de las y los estudiantes dentro de los colegios, debe incorporarse como parte del proceso de estimación de los parámetros del modelo (Hox, 2010).

De acuerdo con el enfoque multigrupo se estimaron los modelos multinivel para cada uno de las dos categorías de población, colegios de territorios indígenas y colegios del GAM, cuyos resultados se muestran en la Tabla 2. 
TABLA 2

Análisis Multigrupo: Resultados de los modelos multinivel para predecir el Contador de Rezago en Asignaturas de Contenido Matemático y el Haber Presentado en Matemática en los 13 colegios de la muestra. $\mathrm{N}=356$, mínimo de observaciones por colegio $=5$

\begin{tabular}{|c|c|c|c|c|c|}
\hline \multirow[b]{2}{*}{ Predictores } & \multicolumn{3}{|c|}{$\begin{array}{l}\text { Contador de rezago en asignaturas de } \\
\text { contenido Matemático }\end{array}$} & \multicolumn{2}{|c|}{$\begin{array}{l}\text { ¿Realizó pruebas de aplazados } \\
\text { en matemática? }\end{array}$} \\
\hline & $\begin{array}{l}\text { Coeficiente } \\
\text { original }\end{array}$ & $\begin{array}{l}\text { Coeficiente } \\
\text { estandarizado }\end{array}$ & Valor $p$ & Odds ratio & Valor $p$ \\
\hline & TERRITORI & DÍGENAS & & & \\
\hline SEXO & $-0,082$ & $-0,063$ & 0,382 & 0,683 & 0,454 \\
\hline $\mathrm{EDAD}$ & 0,124 & 0,047 & 0,368 & 1,054 & 0,457 \\
\hline AUTOEFICACIA & $-0,209$ & $-0,247$ & 0,002 & 0,268 & 0,001 \\
\hline UTILIDAD & 0,057 & 0,079 & 0,261 & 1,048 & 0,860 \\
\hline BENEVOLENTE & 0,054 & 0,069 & 0,566 & 0,761 & 0,609 \\
\hline HOSTIL & $-0,062$ & $-0,072$ & 0,387 & 1,239 & 0,602 \\
\hline DOMINIO & 0,026 & 0,035 & 0,688 & 0,875 & 0,708 \\
\hline EQUIDAD & 0,062 & 0,063 & 0,356 & 1,048 & 0,901 \\
\hline CONS & 0,467 & $-0,065$ & 0,68 & 24,918 & 0,263 \\
\hline$\overline{R^{\wedge} 2}$ & $8,04 \%$ & & & $6,85 \%$ & \\
\hline \multirow[t]{2}{*}{ Rho } & 0,16 & & & 0,28 & \\
\hline & $G A M$ & & & & \\
\hline$\overline{\text { SEXO }}$ & $-0,048$ & $-0,037$ & 0,623 & 0,686 & 0,293 \\
\hline $\mathrm{EDAD}$ & 5,434 & 0,324 & 0,000 & 2,495 & 0,001 \\
\hline AUTOEFICACIA & $-0,224$ & $-0,264$ & 0,001 & 0,399 & 0,000 \\
\hline UTILIDAD & $-0,044$ & $-0,061$ & 0,483 & 1,022 & 0,923 \\
\hline BENEVOLENTE & 0,114 & 0,146 & 0,114 & 1,634 & 0,068 \\
\hline HOSTIL & 0,013 & 0,015 & 0,871 & 1,046 & 0,884 \\
\hline DOMINIO & 0,039 & 0,052 & 0,663 & 0,934 & 0,830 \\
\hline EQUIDAD & 0,161 & 0,163 & 0,099 & 1,21 & 0,587 \\
\hline CONS & $-14,904$ & 0,144 & 0,000 & $4.20 e-07$ & 0,003 \\
\hline$\overline{R^{\wedge} 2}$ & $18,62 \%$ & & & $18,00 \%$ & \\
\hline Rho & 0,00 & & & 0,00 & \\
\hline
\end{tabular}

Fuente: Elaboración propia

Nota: Sexo: 1 si el estudiantado es mujer, 0 si es hombre

Reprobó Matemática: 1 Sí, 0 No

Contador de Rezago: Rango de 0 a 4

En cuanto a las dos variables sociodemográficas, sexo y edad, la evidencia mostrada en la Tabla 2 apunta a que la edad, en el GAM y, como era de esperar, representa una variable de control muy relevante, dado que está asociada con los indicadores de rezago, tanto para la variable métrica, como para la variable dicotómica. Por otra parte, en ninguna de las dos poblaciones se presentaron diferencias de relevancia en cuanto al sexo. Este resultado contradice hallazgos de investigaciones en torno a menores rendimientos para las mujeres en matemática. Se prefiere no especular sobre posibles razones para este comportamiento específico en estas muestras. 
En el caso de la variable métrica, contador de rezago específico para asignaturas de contenido matemático, el coeficiente estandarizado de regresión es igual a 0,324; muy superior al umbral mínimo de 0,10 necesario para declarar un resultado como relevante o de importancia práctica. El hecho de que, en este estudio en particular, la mayoría de los valores de p iguales o próximos a 0 son justo los que corresponden a los coeficientes que resultaron de importancia práctica, tiene que ver, precisamente, con las magnitudes de los tamaños de muestra, que están en el rango de los tamaños muestrales donde es frecuente que coincidan el criterio de significancia estadística y el criterio de importancia práctica. Con tamaños de muestra más bajos o altos no se presenta esta coincidencia. Un ejemplo de mucha actualidad son las aplicaciones en Big Data, donde un número tan grande de datos resulta inútil a las pruebas de significancia estadística, pues aún efectos o magnitudes sin ninguna relevancia práctica generan resultados estadísticamente significativos.

En el caso de la muestra de los colegios del GAM la variable dicotómica que identifica si el estudiantado ha realizado la prueba de aplazados por reprobar matemática, el coeficiente odds ratio, que es una medida del riesgo relativo de pertenecer a esa categoría, mostró un valor es 2,495. Esto indica que al aumentar la edad ese riesgo de pertenecer al grupo de los aplazados aumenta más de dos veces. Para los colegios de territorios indígenas la asociación entre el contador de rezago y la edad resulta casi nula, con un coeficiente estandarizado de 0,047 y para la variable dicotómica el odds ratio es prácticamente 1 , lo que indica que no hay diferencias según edad en el grupo de aplazados versus no aplazados.

En lo que toca al grupo de variables actitudinales, la autoeficacia matemática y la utilidad percibida de la Matemática, la primera tuvo un alto poder predictivo en ambas poblaciones, tanto para el contador de rezago como para la variable dicotómica que identifica a las y los estudiantes que debieron presentar las pruebas de aplazados de Matemática. La relación, como predice la teoría, es inversa: a mayor autoeficacia matemática menor rezago. Estos resultados coinciden con numerosas investigaciones realizadas a nivel nacional e internacional, como ya fue discutido en el estado de la cuestión (Kaya y Bozdag, 2016; Olivier et. al., 2019 y Schöber et. al., 2018). También son consistentes con los de otros estudios, en donde se ha controlado por las habilidades básicas de razonamiento del estudiantado (Smith-Castro, Montero-Rojas, Moreira-Mora y Zamora-Araya, 2019). Más aún, a pesar de las diferencias contextuales, el coeficiente estandarizado de esta variable en el modelo para el contador de rezago es muy similar en ambos grupos $(-0.25$ para los colegios de territorios indígenas y -0.26 para los colegios del GAM).

Precisamente, en este caso particular, el valor agregado del hallazgo es que incluye estudiantes de colegios en territorios indígenas, población que hasta este momento no había sido objeto específico de estudios de esta naturaleza. Por otro lado, la escala de utilidad matemática no presenta niveles de asociación relevantes en ninguna de las dos poblaciones.

Por último, en términos de las creencias sociales con relación con el género, se contaba con cuatro escalas: sexismo hostil y sexismo benevolente como indicadores generales, y como indicadores específicos una escala de la matemática como dominio masculino y una escala de equidad de género en el área matemática. De ellas solo resultaron con importancia práctica a nivel predictivo el sexismo benevolente y la escala de equidad para las y los estudiantes de colegios del GAM. Ambas muestran una relación directa con el rezago en este grupo, es decir a mayor sexismo benevolente y a mayor equidad de género en contextos matemáticos, mayor rezago. En el caso de la muestra de colegios de territorios indígenas, ninguna de estas variables de creencias sociales presentó evidencias de relación con los indicadores de rezago, sugiriendo que para esta población particular debe evaluarse la validez de estos instrumentos, así como la posibilidad de que los constructos que se intentan medir puedan estar afectados por diferencias culturales y contextuales particulares a esta población.

Un resultado interesante que lleva a la reflexión es la diferencia en el poder predictivo de las variables, según se trate del grupo de colegios del GAM o el grupo de colegios de territorios indígenas, pues el indicador de varianza explicada por el modelo ( $\mathrm{R}^{\wedge} 2$ en la Tabla 2) en el caso del contador de rezago es sólo $8 \%$ para los colegios de territorios indígenas, mientras que es $19 \%$ para los colegios del GAM. Asimismo, para el indicador dicotómico que identifica a los aplazados en matemática las variables del modelo solo explican $8 \%$ de la 
variabilidad en los colegios de territorios indígenas, comparada con 18\% para los de GAM. Estos resultados sugieren que es necesario explorar otros factores en el contexto específico de los colegios de territorios indígenas para poder explicar mejor la variabilidad en los indicadores de rezago, más allá de los que se han identificado en este estudio.

Por último, los valores del coeficiente Rho en la Tabla 2 se refieren al grado de correlación intraclase que se evidencia para los conglomerados, es decir, para los colegios, en cada uno de los dos grupos. En el caso de los colegios de territorios indígenas existe un nivel de correlación intraclase relativamente alto para las dos variables dependientes, sobre todo para la variable dicotómica que identifica a los que han presentado la prueba de aplazados en matemática, dado su valor de 0,28 . Esto significa que las diferencias entre los colegios de territorio indígena explican parcialmente la variabilidad en los niveles de esta variable. Por el contrario, en el caso de los colegios del GAM, el valor de Rho es tan pequeño que se registra como igual a cero en la Tabla 2 , de tal forma que se concluye que no hay diferencias de importancia, a nivel de los colegios, en términos de explicar el contador de rezago y el indicador de aplazados.

\section{Conclusiones}

Se trata de uno de los primeros esfuerzos investigativos que se realiza en Costa Rica para estimar factores asociados al rezago en colegios públicos que están ubicados en territorios indígenas, comparados con colegios públicos del GAM.

En el grupo de las variables sociodemográficas, el sexo no brinda, para ninguno de los dos grupos, evidencia de una relación de importancia con las variables de rezago en Matemática o en asignaturas con ese contenido. Mientras que la evidencia de edad es contundente sobre su relación con el rezago en la muestra de colegios del GAM, pero no para los colegios de territorios indígenas. Sin embargo, se considera que, en estudios posteriores con este grupo de población, deben seguirse incluyendo estas variables, al representar la edad una variable de control, y, en el caso del sexo, existir mucha evidencia previa de desventajas para las mujeres.

En lo referente a las actitudes hacia la matemática, la conclusión más relevante a partir del análisis es la considerable importancia, en ambas poblaciones, de la autoeficacia matemática, como predictora para los dos indicadores de rezago. A partir de este resultado, una recomendación directa es fomentar la autoeficacia matemática en los dos grupos de población estudiantil; por ello, es necesario que en los objetivos de aprendizaje desarrollados por el Ministerio de Educación se incluya la promoción de la autoeficacia matemática, y otras variables socioemocionales, como parte de la mediación pedagógica. Por el contrario, el otro componente de la dimensión de actitudes, la escala de utilidad percibida de la Matemática, no presenta ninguna relación de importancia con el rezago. Una explicación para este resultado es que la escala se enfoca en la utilidad de la matemática para un futuro, no da importancia a su papel como herramienta para afrontar adecuadamente la vida cotidiana; por lo que futuras investigaciones podrían explorar el significado y valor de la matemática para estudiantes y docentes.

En cuanto a las creencias sociales en torno al género, tanto en general como en contextos matemáticos, y particularmente para los colegios ubicados en el GAM, la escala de sexismo benevolente y una de las escalas de estereotipos de género en contexto matemático presentaron una asociación directa de cierta relevancia con el contador de rezago. Se trata, de resultados emergentes para los cuales aún no existe una teoría consolidada y deben ser explorados en investigaciones futuras. Una de las razones que podría explicar la no relevancia del sexismo hostil es que el contenido de la escala refiere más a situaciones adultas, poco familiares para los adolescentes. En cuanto a la escala de equidad los resultados van en dirección contraria a lo esperado según la teoría, por lo que debería investigarse con más profundidad en nuevos estudios. También llama la atención que la escala de dominio masculino de la matemática no generara evidencia predictiva, dado que, en contextos internacionales y en este tipo de poblaciones, ha resultado de importancia. 
Otra conclusión de importancia, a partir del bajo poder predictivo de los modelos para las y los estudiantes de colegios de territorios indígenas, es que se deben realizar más estudios específicos con esta población para identificar otros factores de relevancia, más allá de los incluidos aquí, que puedan contribuir a comprender mejor el fenómeno del rezago en este contexto particular.

\section{Referencias Bibliográficas}

Aponte-Hernández, E. (2008). Desigualdad, inclusión y equidad en la educación superior en américa latina y el caribe: tendencias y escenario alternativo en el horizonte 2021. En Ana Lúcia Gazzola y Axel Didriksson (Eds.) Tendencias de la Educación Superior en América Latina y el Caribe (pp.113-151). Venezuela: UNESCO. Recuperado de https://bit.ly/3exrzee

Bong, M. (2001). Role of Self-Efficacy and Task-Value in Predicting College Students' Course Performance and Future Enrollment Intentions. Contemporary educational psychology, 26(4), 553-570. doi: https://doi.org/10.1006/c eps.2000.1048

Brown, C. y Leaper, C. (2010). Latina and European American girls' experiences with academic sexism and their selfconcepts in mathematics and science during adolescence. Sex Roles, 63, 860-870. doi: https://doi.org/10.1007 /s11199-010-9856-5

Cárdenas, M., Lay, L., González, C., Calderón, C. y Alegría, I. (2010). Inventario de sexismo ambivalente: adaptación, validación y relación con variables psicosociales. Salud \& Sociedad, 1(2), 125-135. Recuperado de https://dialn et.unirioja.es/servlet/articulo?codigo $=4016898$

Carvajal-Jiménez, V., Cubillo-Jiménez, K. y Vargas-Morales, M. (2017). Poblaciones indígenas de Costa Rica y su acceso a la educación superior. División de Educación Rural: Una alternativa de formación. Revista EDUCARE, 21(3), 1-31. doi: https://doi.org/10.15359/ree.21-3.21

Casis, M., Rico, N. y Castro, E. (2017). Motivación, autoconfianza y ansiedad como descriptores de la actitud hacia las matemáticas de los futuros profesores de educación básica de Chile. PNA, 11(3), 181-203. Recuperado de $\mathrm{h}$ ttp://hdl.handle.net/10481/45499

Cervini, R. (2010). El 'efecto escuela' en la educación primaria y secundaria: El caso de Argentina. Revista Iberoamericana sobre Calidad, Eficacia y Cambio en Educación, 8(1), 7-25. Recuperado de https://revistas.uam .es/index.php/reice/article/view/5372

Coleman, J.S. (1966). Equality of educational opportunity. Estados Unidos: National Center for Educational Statistics. Recuperado de https://files.eric.ed.gov/fulltext/ED012275.pdf

Cornejo, R. y Redondo, J.M. (2007). Variables y factores asociados al aprendizaje escolar. Una discusión desde la investigación actual. Estudios Pedagógicos, 33(2), 155-175. Recuperado de https://scielo.conicyt.cl/pdf/estped /v33n2/art09.pdf

Eccles, J. S. y Wigfield, A. (2002). Motivational beliefs, values, and goals. Annual Review of Psychology, 53, 109-132. doi: https://doi.org/10.1146/annurev.psych.53.100901.135153

Ellis, J., Fosdick, B.K. \& Rasmussen, C. (2016). Women 1.5 Times More Likely to Leave STEM Pipeline after Calculus Compared to Men: Lack of Mathematical Confidence a Potential Culprit. PLOS ONE Journal, 11(7). doi: ht tps://doi.org/10.1371/journal.pone.0157447

Fennema, E. y Sherman, J. (1976). Fennema-Sherman Mathematics Attitudes Scales: Instruments Designed to Measure Attitudes toward the Learning of Mathematics by Females and Males. Journal for Research in Mathematics Education, 7(5), 324-326. doi: https://doi.org/10.2307/748467

Fernández, A. (2018). Estadísticas del subsistema de educación Indígena de Costa Rica. Historia y situación actual (1800 - 2016). Costa Rica: Ministerio de Educación Pública. Recuperado de https://www.mep.go.cr/indicadores_ed u/BOLETINES/ind.pdf

Fuentes, E. (2011). Características demográficas y socioeconómicas de las poblaciones indígenas de Costa Rica (Censo 2011). Costa Rica: Instituto Nacional de Estadística y Censos. Recuperado de https://www.inec.cr/sites/defau lt/files/documentos/inec_institucional/publicaciones/anpoblaccenso2011-04.pdf.pdf 
Tania Elena Moreira-Mora, et al. Rezago en asignaturas de contenido matemático en estudiantes de ú...

Gamazo, A., Martínez-Abad, F., Olmos-Miguelañez, S. y Rodríguez-Conde, M.J. (2018). Evaluación de factores relacionados con la eficacia escolar en PISA 2015. Un análisis multinivel. Revista de Educación, (379), 52-78. doi: https://doi.org/10.4438/1988-592X-RE-2017-379-369

Galindo, J. (6 diciembre de 2019). La desigualdad latinoamericana empieza en la escuela. El país. Recuperado de http s://elpais.com/internacional/2019/12/05/america/1575575380_272998.html

Glick, P. y Fiske. S.T. (1996). The Ambivalent Sexism Inventory: Differentiating hostile and benevolent sexism. Journal of Personality and Social Psychology, 70(3), 491-512. doi: https://doi.org/10.1037/0022-3514.70.3.491

Glick, P. y Fiske, S. T. (2011). Ambivalent sexism revisited. Psychology of Women Quarterly, 35(3), 530-535. doi: ht tps://doi.org/10.1177/0361684311414832

Glick, P., Wilkerson, M. y Cuffe, M. (2015). Masculine Identity, Ambivalent Sexism, and Attitudes Toward Gender Subtypes. Social Psychology, 46(4), 210-217. doi: https://doi.org/10.1027/1864-9335/a000228

Hyde, J. S., Lindberg, S. M., Linn, M. C., Ellis, A. B. y Williams, C. C. (2008). Gender similarities characterize math performance. Science, 321(5888), 494-495. doi: https://doi.org/10.1126/science.1160364

Hox, J. (2010). Multilevel Analysis: Techniques and Applications. New York, USA: Routledge.

Instituto Nacional de las Mujeres (2006). Las mujeres indígenas: Estadísticas de la Exclusión. Costa Rica: Instituto Nacional de las Mujeres. Recuperado de https://bit.ly/2DFjLud

Instituto Nacional de Estadística y Censos [INEC] (2013). X Censo Nacional de Población y VI de Vivienda 2011: Territorios Indigenas. Principales indicadores demográficos y socioeconómicos. Costa Rica: Instituto Nacional de Estadística y Censos. Recuperado de https://www.inec.go.cr/documento/censo-2011-territorios-indigenas-pri ncipales-indicadores-demograficos-y-socioeconomicos

Jacobs, J. E. y Eccles, J. S. (1992). The impact of mothers' gender-role stereotypic beliefs on mothers' and children's ability perceptions. Journal of Personality and Social Psychology, 63(6), 932-944. doi: https://doi.org/10.1037 /0022-3514.63.6.932

Kaya, D. y Bozdag, H. C. (2016). Resources of Mathematics Self-Efficacy and Perception of Science Self-Efficacy as Predictors of Academic Achievement. European Journal of Contemporary Education, 18(4), 438-451. doi: http s://doi.org/10.13187/ejced.2016.18.438

Lindberg, S. M., Hyde, J. S., Petersen, J. L. y Linn, M. C. (2010). New trends in gender and mathematics performance: a meta-analysis. Psychological bulletin, 136(6), 1123-1135. doi: https://doi.org/10.1037/a0021276

Little, T.D. (2013). Longitudinal Structural Equation Modeling. New York, USA: The Guilford Press.

Martínez, C. y Murillo, F. J. (2016). Investigación iberoamericana sobre enseñanza eficaz. Revista mexicana de investigación educativa, 21(69), 471-499. Recuperado de https://dialnet.unirioja.es/servlet/articulo?codigo=56 14914

Montero-Rojas, E., Castelain, T., Moreira-Mora, T. E., Alfaro-Rojas, L., Cerdas-Núñez, D., García-Segura, A. y Roldán-Villalobos, M.G. (2013). Evidencias iniciales de validez de criterio de los resultados de una Prueba de razonamiento con figuras para la selección de estudiantes indígenas para la Universidad de Costa Rica y el Instituto Tecnológico de Costa Rica. Revista Educación, 37(2), 103-117. doi: https://doi.org/10.15517/reved u.v37i2.12928

Muñoz, J., Arias, M.A. y Mato, M. (2018). Elementos predictores del rendimiento matemático en estudiantes de Educación Secundaria Obligatoria. Profesorado. Revista de Curriłtulum y Formación de Profesorado, 22 (3), 391-413. doi: https://doi.org/10.30827/profesorado.v22i3.8008

Murillo, F. J. y Román, M. (2011). ¿La escuela o la cuna? Evidencias sobre su aportación al rendimiento de los estudiantes de América Latina. Estudio multinivel sobre la estimación de los efectos escolares. Profesorado Revista de Curriculum y Formación de Profesorado, 15(3), 27-50. Recuperado de http://www.ugr.es/ recfpro/rev153A RT3.pdf

Olivier, E., Archambault, I., De Clercq, M. y Galand, B. (2019). Student self-efficacy, classroom engagement, and academic achievement: comparing three theoretical frameworks. Journal of youth and adolescence, 48, 326-340. doi: https://doi.org/10.1007/s10964-018-0952-0 
Organización para la Cooperación y el Desarrollo Económicos (OCDE). (2015). The ABC of Gender Equality in Education: Aptitude, Behaviour, Confidence. París, Francia: OECD Publishing. doi: http://dx.doi.org/10.1787 /9789264229945-en

Organización para la Cooperación y el Desarrollo Económicos (OCDE). (2016). PISA 2015 Results (Volume I): Excellence and Equity in Education. París, Francia: OECD Publishing. doi: https://doi.org/10.1787/9789264 266490-en

Palacios, A., Arias, V. y Arias, B. (2014). Las actitudes hacia las matemáticas: construcción y validación de un instrumento para su medida. Revista de psicodidáctica, 19(1), 67-91. Recuperado de https://ojs.ehu.eus/index.p $\mathrm{hp} / \mathrm{psicodidactica/article/download/8961/9943}$

Programa Estado de la Nación (2017). Sexto informe estado de la educación. Recuperado de https://www.estadonacio n.or.cr/educacion2017/assets/ee6-informe-completo.pdf

Pennington, C. R., Heim, D., Levy, A. R. y Larkin, D. T. (2016). Twenty Years of Stereotype Threat Research: A Review of Psychological Mediators. PLOS ONE 11(1), 1-25. doi: https://doi.org/10.1371/journal.pone.0146 487

Reilly, D., Neumann, D. L. y Andrews, G. (2019). Investigating Gender Differences in Mathematics and Science: Results from the 2011 Trends in Mathematics and Science Survey. Research in Science Education, 49, 25-50. doi: https://doi.org/10.1007/s11165-017-9630-6

Schöber, C., Schütte, K., Köller, O., McElvany, N. \& Gebauer, M. M. (2018). Reciprocal effects between self-efficacy and achievement in mathematics and reading. Learning and Individual Differences, 63, 1-11. doi: https://doi.o $\mathrm{rg} / 10.1016 / \mathrm{j}$. lindif.2018.01.008

Schwarzer, R., Bäßler, J., Kwiatek, P., Schröder, K. y Zhang, J. X. (1997). The assessment of optimistic self-beliefs: Comparison of the German, Spanish, and Chinese versions of the General Self-efficacy Scale. Applied Psychology: An International Review, 46(1), 69-88. doi: https://doi.org/10.1111/j.1464-0597.1997.tb01096.x

Smith, V. y Molina, M. (2011). La entrevista cognitiva: Guía para su aplicación en la evaluación y mejoramiento de instrumentos de papel y lápiz. Cuadernos Metodológicos, 5, 1-114. Recuperado de http://iip.ucr.ac.cr/sites/defa ult/files/contenido/Entrevista\%20Cognitiva\%20\%282011\%29.pdf

Smith-Castro, V., Montero-Rojas, E., Moreira-Mora, T. E. y Zamora-Araya, J. A. (2019). Expected and unexpected effects of sexism on women's mathematics performance. Revista Interamericana de Psicología, 53(1), 28-44. Recuperado de https://journal.sipsych.org/index.php/IJP/article/view/905

Spencer, J., Steele, M. y Quinn, M. (1999). Stereotype Threat and Women's Math Performance. Journal of Experimental Social Psychology, 35(1), 4-28. doi: https://doi.org/10.1006/jesp.1998.1373

UNESCO-OREALC (2016). Recomendaciones de politicas educativas en América Latina en base al TERCE. Santiago, Chile: UNESCO. Recuperado de http://repositorio.minedu.gob.pe/handle/123456789/4513

Walton, G. M. y Spencer, S. J. (2009). Latent ability: Grades and test scores systematically underestimate the intellectual ability of negatively stereotyped students. Psychological Science, 20(9), 1132-1139. doi: https://doi .org/10.1111/j.1467-9280.2009.02417.x

Wigfield, A. y Gladstone, J. R. (2019). What Does Expectancy-value Theory Have to Say about Motivation and Achievement in Times of Change and Uncertainty? En E. N. Gonida y M. S. Lemos (Eds), Motivation in Education at a Time of Global Change: Theory, Research, and Implications for Practice (pp 15-32). doi: https:// doi.org/10.1108/S0749-742320190000020002

Woitschach, P., Fernández-Alonso, R., Martínez-Arias, R. y Muñiz, J. (2017). Influencia de los centros escolares sobre el rendimiento académico en Latinoamérica. Revista de Psicología y Educación, 12(2), 138-154. Recuperado de http://www.revistadepsicologiayeducacion.es/pdf/152.pdf

Zamora-Araya. A. (2020). Las actitudes hacia la matemática, el desarrollo social, el nivel educativo de la madre y la autoeficacia como factores asociados al rendimiento académico en la matemática. Uniciencia, 34(1), 74-87. doi: https://doi.org/10.15359/ru.34-1.5 
Tania Elena Moreira-Mora, et al. Rezago en asignaturas de contenido matemático en estudiantes de ú...

Zell, E., Strickhouser, J.E., Lane, T.N. y Teeter, S.R. (2016). Mars, Venus, or Earth? Sexism and the exaggeration of psychological gender differences. Sex Roles: A Journal of Research, 75, 287-300. doi: https://doi.org/10.1007/s 11199-016-0622-1

Zorrilla, M. (2009). ¿Cuál es la aportación de la escuela secundaria mexicana en el rendimiento de los alumnos en Matemáticas y Español? Revista electrónica de investigación educativa, 11(2), 1-29. Recuperado de https://redie .uabc.mx/redie/article/view/236

\section{Notas}

[1] Nota: Se agradece al Consejo Nacional de Rectores (CONARE) por el financiamiento a esta investigación. También un profundo agradecimiento a los colegios públicos que tuvieron a bien abrir sus puertas para recolectar la información de sus estudiantes.

\section{BY-NC-ND}

